\title{
Scalable Integration of Long-Lived Quantum Memories into a Photonic Circuit
}

\author{
Sara L. Mouradian, ${ }^{1, *}$ Tim Schröder, ${ }^{1}$ Carl B. Poitras, ${ }^{2}$ Luozhou Li,${ }^{1}$ Jordan Goldstein, ${ }^{1}$ Edward H. Chen, ${ }^{1}$ Michael Walsh, ${ }^{1}$ \\ Jaime Cardenas, ${ }^{2}$ Matthew L. Markham, ${ }^{3}$ Daniel J. Twitchen, ${ }^{3}$ Michal Lipson, ${ }^{2, \dagger}$ and Dirk Englund ${ }^{1, \$}$ \\ ${ }^{1}$ Department of Electrical Engineering and Computer Science, Massachusetts Institute of Technology, \\ Cambridge, Massachusetts 02139, USA \\ ${ }^{2}$ School of Electrical and Computer Engineering, Cornell University, Ithaca, New York 14853, USA \\ ${ }^{3}$ Element Six, 3901 Burton Drive, Santa Clara, California 95054, USA
}

(Received 23 August 2014; revised manuscript received 15 April 2015; published 21 July 2015)

\begin{abstract}
We demonstrate a photonic circuit with integrated long-lived quantum memories. Precharacterized quantum nodes-diamond microwaveguides containing single, stable, negatively charged nitrogenvacancy centers-are deterministically integrated into low-loss silicon nitride waveguides. These quantum nodes efficiently couple into the single-mode waveguides with $>1 \mathrm{Mcps}$ collected into the waveguide, have narrow single-scan linewidths below $400 \mathrm{MHz}$, and exhibit long electron spin coherence times up to $120 \mu \mathrm{s}$. Our system facilitates the assembly of multiple quantum nodes with preselected properties into a photonic integrated circuit with near unity yield, paving the way towards the scalable fabrication of quantum information processors.
\end{abstract}

DOI: 10.1103/PhysRevX.5.031009

Advanced quantum information systems, such as quantum computers [1] and quantum repeaters [2], require multiple entangled quantum memories that can be individually controlled [3]. Over the past decade, there has been rapid theoretical and experimental progress in developing such entangled networks using stationary quantum bits (qubits) connected via photons [4-6]. Photonic integrated circuits (PICs) could provide a compact, phase-stable, and scalable architecture for such quantum networks. However, the realization of this promise requires near-unity-yield fabrication of high-quality solid-state quantum memories efficiently coupled to low-loss single-mode waveguides.

A promising solid-state quantum memory with secondscale spin coherence times is the negatively charged nitrogen-vacancy $(\mathrm{NV})$ center in diamond $[7,8]$. Its electronic spin state can be optically initialized, manipulated, measured $[9,10]$, and mapped onto nearby auxiliary nuclear memories [11], which allows for quantum error correction [12]. Quantum network protocols based on these unique qualities have been proposed [13,14], and entanglement generation and state teleportation between two spatially separated quantum memories have been demonstrated $[15,16]$. Translating such entanglement techniques into an on-chip architecture promises scalability, but can only

\footnotetext{
*smouradi@mit.edu

†m1292@cornell.edu

*englund@mit.edu
}

Published by the American Physical Society under the terms of the Creative Commons Attribution 3.0 License. Further distribution of this work must maintain attribution to the author(s) and the published article's title, journal citation, and DOI.
Subject Areas: Photonics, Quantum Information

succeed if an efficient photonic architecture can be fabricated with a high yield. So far, waveguide patterning in diamond is challenging, preventing low-loss waveguides in the optical domain around $637 \mathrm{~nm}$, the zero phonon line (ZPL) of the NV. This is in contrast to silicon nitride (SiN)-based photonics, which relies on well-developed fabrication processes [17], is CMOS compatible [18], and has a large band gap $(\sim 5 \mathrm{eV})$ and high index of refraction $(n=2.1)$, which makes it ideal for routing the visible emission of NVs.

A second challenge in creating a scalable solid-state quantum network architecture is the inherently low yield production of high-quality quantum nodes due to the stochastic process of NV creation. The number of fabrication attempts necessary to create a monolithic network in which all nodes contain NV quantum memories with the desired spectral and spin properties scales exponentially with the number of desired nodes, which is prohibitive with current NV creation yields of well less than $10 \%[19,20]$. Thus, while proof-of-principle network components have been demonstrated [21-23], a method for the scalable fabrication of quantum networks has not yet been shown.

Here, we address the challenge of fabricating a scalable quantum network architecture with a hybrid approach [24-28] in which quantum nodes based on NV centers are precharacterized and integrated into SiN PICs that can be fabricated with near-unity yield. The preselection step guarantees that every node in the PIC contains a NV with the desired spectral and spin properties, and is well coupled to the optical mode. With this bottom-up approach, the number of fabrication attempts necessary to create a quantum network scales linearly with the number of desired nodes. 
In our approach, each quantum node consists of an NV in a diamond microwaveguide $(\mu \mathrm{WG})$ suspended over a coupling region in a $\mathrm{SiN}$ waveguide, as illustrated in Fig. 1(a). The diamond $\mu \mathrm{WG}$ supports single-mode propagation over the $\mathrm{NV}$ emission spectrum with a cross section of $200 \times 200 \mathrm{~nm}$. Suspending it across an air gap in the SiN WG enables efficient coupling of the NV to the single optical mode in the diamond $\mu \mathrm{WG}$. Finite-difference time-domain (FDTD) simulations show that with this air gap up to $86 \%(78 \%)$ of the NV zero phonon line (phonon side band) fluorescence intensity at $\lambda=638 \mathrm{~nm}$ $(\lambda=600-780 \mathrm{~nm})$ is guided into the diamond $\mu \mathrm{WG}$ with optimal NV position (the mode maximum of the diamond $\mu \mathrm{WG}$ ), as seen in Fig. 1(b) (dashed curve).

The SiN waveguide also supports single-mode propagation over the $\mathrm{NV}$ emission spectrum with a cross section of $400 \times 400 \mathrm{~nm}$. Tapering of the overlapping SiN and diamond regions allows for an approximately adiabatic transition between the diamond and $\mathrm{SiN}$ waveguide modes. We perform FDTD simulations to optimize the tapered regions of the diamond and $\mathrm{SiN}$ waveguides to maximize the coupling between the diamond and $\mathrm{SiN}$ waveguides, as shown in Fig. 1. Our simulations indicate that up to $96 \%$ of the NV fluorescence emitted into the diamond waveguide is transferred into the $\mathrm{SiN}$ waveguide with the optimized overlapping regions shown in Fig. 1(c) (see Appendix C). Thus, as shown in Fig. 1(b) (solid curve), we estimate that the total collection efficiency from the NV ZPL through the diamond $\mu \mathrm{WG}$ into the SiN WG is $83 \%$ for the optimized design shown in Fig. 1(c).

We fabricate the diamond $\mu$ WGs from a 200 -nm-thick single-crystal diamond membrane which was thinned from a 5- $\mu \mathrm{m}$ diamond slab produced by chemical vapor deposition. Natural NVs occur at a concentration of approximately $0.25 \mathrm{NV} / \mu \mathrm{m}^{3}$. A patterned silicon membrane is used as a hard etch mask [29,30] during an oxygen plasma etch of the diamond membrane [31]. We fabricate the SiN waveguides from a 400-nm-thick SiN layer deposited on silicon dioxide [17]. The waveguides are cladded with a
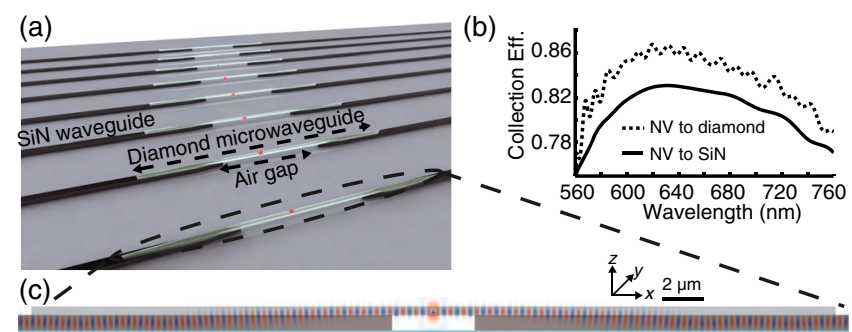

FIG. 1. (a) Sketch of a SiN PIC with multiple quantum nodes. (b) Simulation of the collection efficiency of NV fluorescence into the diamond $\mu \mathrm{WG}$ (dashed line) and into the $\mathrm{SiN}$ single-mode waveguide with optimal parameters (solid line). (c) FDTD simulation ( $E_{x}$ field) showing the mode transfer from a single-mode diamond waveguide into a single-mode SiN waveguide.
3- $\mu \mathrm{m}$ layer of $\mathrm{SiO}_{2}$ except for a $50-\mu \mathrm{m}$ window over the coupling region for the integration of the diamond $\mu \mathrm{WGs}$ shown in Fig. 2(b) (see Appendix A for more fabrication details).

The SEM in Fig. 2(b) shows a typical array of diamond $\mu$ WGs. In this experiment, we use 12- $\mu$ m-long, 200-nmwide $\mu$ WGs with $4-\mu \mathrm{m}$-long tapers down to about $100 \mathrm{~nm}$ on either side. This minimum taper size is larger than optimal, but the fabrication yield is significantly increased compared to thinner ends. FDTD simulations indicate that this geometry should yield a $42.5 \%$ coupling efficiency from the NV ZPL to the SiN waveguide (see Appendix C).

To demonstrate the scalability of our approach and the high optical coupling that is possible with the waveguide geometry, we use photoluminescence (PL) confocal raster scans (N.A. $=0.9,532 \mathrm{~nm}$ excitation), as in Fig. 2(c), to identify and characterize four $\mu \mathrm{WGs}$ with single NVs near the center. The preselected diamond $\mu \mathrm{WGs}$ are picked and transferred onto four consecutive coupling regions of WGs of the same design in the SiN PIC (see Appendix B for transfer details and images). Figures 2(d)

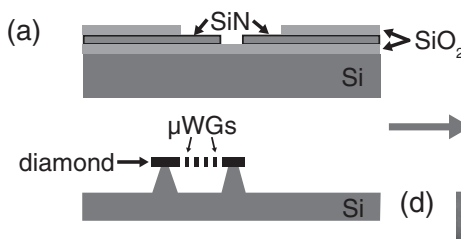

(b)

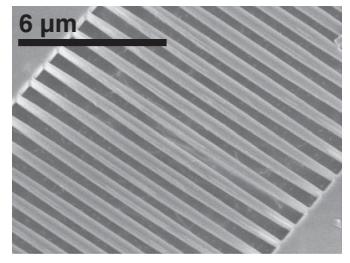

(c)
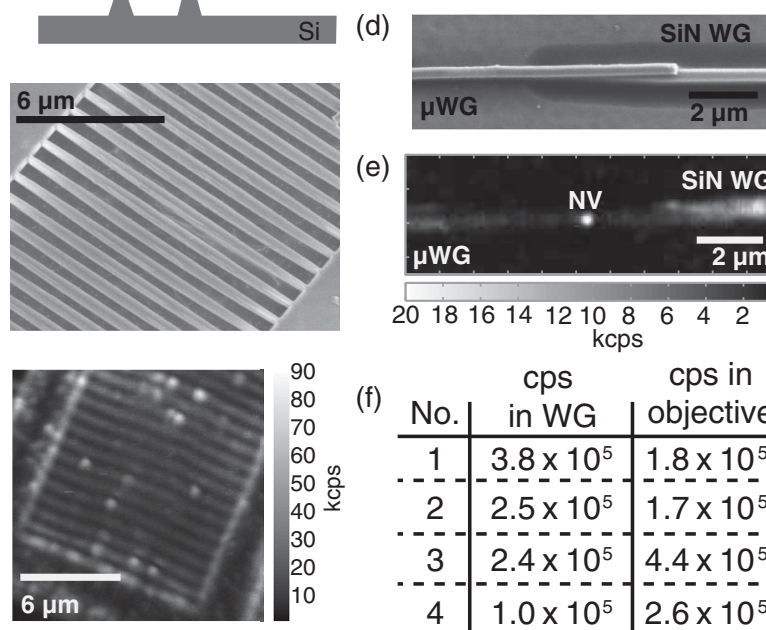

(e)

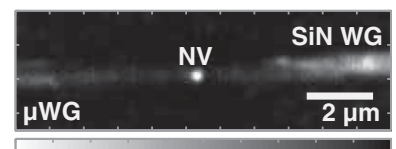
$201816141210 \quad 8 \quad 6 \quad 4 \quad 2 \quad 0$

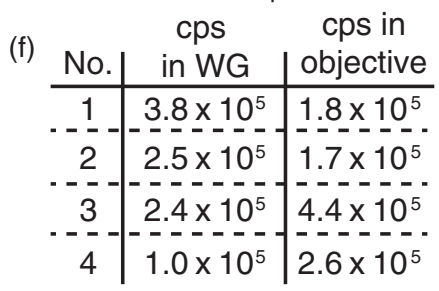

FIG. 2. (a) Illustration of integration of a diamond quantum node into a SiN PIC. Top left: A cross-section view of the coupling region of a SiN PIC before integration of the diamond. Bottom left: An array of diamond $\mu \mathrm{WGs}$ resting on an etched $\mathrm{Si}$ chip. Right: The integration of a diamond $\mu \mathrm{WG}$ onto a coupling region of the SiN PIC. (b) SEM and (c) confocal PL raster scan of a diamond $\mu \mathrm{WG}$ array. (d) SEM and (e) confocal PL raster scan of a diamond $\mu \mathrm{WG}$ placed over a gap in a SiN WG, showing both the gap and the waveguide used to collect the signal. The confocal scan shows the bright NV center in the diamond $\mu \mathrm{WG}$. (f) $\mathrm{NV}$ photons collected into the waveguide and objective for four $\mu \mathrm{WG}$ integrated into consecutive $\mathrm{SiN}$ WGs at $100 \mu \mathrm{W}$ of excitation. 
and 2(e) show a SEM of one of the transferred structures and the corresponding PL scan, respectively.

We evaluate the coupling efficiency into the SiN PIC from each $\mu \mathrm{WG}$ by confocally exciting the single NV from the top and collecting the fluorescence both through the objective and through the SiN waveguide through a lensed single-mode fiber. In both cases, the laser is attenuated with a 550-nm long-pass filter. The table in Fig. 2(f) summarizes the photon count rates obtained for the two collection channels for a 532-nm pump power of $100 \mu \mathrm{W}$. The emission rate into the waveguide is consistently high with variation likely due to differences in the $\mu \mathrm{WG}$ placement, fabrication imperfections, and NV position and dipole orientation.

In the following, we focus on integrated system 1 , which is seen in Figs. 2(d) and 2(e). Figures 3(a) and 3(b) show PL raster scans of the $\mathrm{NV}$ under confocal excitation with confocal and waveguide collection, respectively.

The second-order autocorrelation of photons collected via the confocal setup confirms the single photon emission expected from single NVs, with antibunching as low as $g^{(2)}(0)=0.07$ (see Appendix D). This low background shows that the NVs are extremely well isolated from background sources of fluorescence. Figure 3(c) shows the normalized autocorrelation measurement of the $\mathrm{NV}$ in Figs. 3(a) and 3(b), with $g^{(2)}(0)=0.17$ at $100 \mu \mathrm{W}$ of $532 \mathrm{~nm}$ excitation. We also perform cross-correlation measurements between photons collected via the confocal and waveguide setups. The normalized histogram in Fig. 3(d) indicates clear antibunching at $60 \mu \mathrm{W}$ of excitation with $g^{(2)}(0)=0.48$. The increase in $g^{(2)}(0)$ relative to the confocal autocorrelation measurement is attributed to the generation of background fluorescence due to scattering of the excitation laser into the SiN WG.

Figure 3(e) plots the spectrum of the fluorescence collected through the waveguide including both NV fluorescence and the unwanted PL due to the SiN WG. An interference pattern is visible, which we attribute to an etalon effect at the diamond end facets. This etalon effect indicates an intensity reflection of $r^{2}=0.17$ and a waveguide effective index of 1.65 for the $12-\mu \mathrm{m}$-long diamond $\mu \mathrm{WG}$, which matches the theoretical expectations (see Appendix E).

To evaluate the enhancement in collection efficiency through the waveguide, we perform emitter saturation measurements with both confocal and waveguide collection, as seen in Fig. 3(f). In each case, the excitation polarization is tuned to maximize the signal-to-noise ratio. For confocal collection, this entails maximizing the NV excitation rate, while under waveguide collection, this entails limiting the coupling of the $532 \mathrm{~nm}$ excitation into the waveguide to minimize background fluorescence. The optimized polarization for waveguide collection reduces the NV excitation rate, and as such increases the saturation intensity from $135 \mu \mathrm{W}$ via confocal collection to $350 \mu \mathrm{W}$ via waveguide collection. For confocal (waveguide) collection, $16 \mathrm{kcps}(55 \mathrm{kcps})$ were detected at $60 \mu \mathrm{W}$ of excitation (with polarization optimized for waveguide signal-to-noise ratio), as used to measure the crosscorrelation function seen in Fig. 3(d).

Figure 3(g) shows the fits in Fig. 3(f) without the linear background terms, and corrected for the measured collection efficiencies of each collection pathway. The efficiency

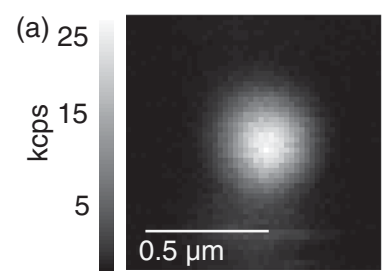

(b) 35

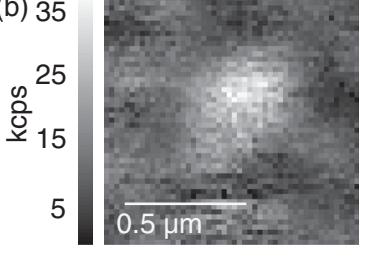

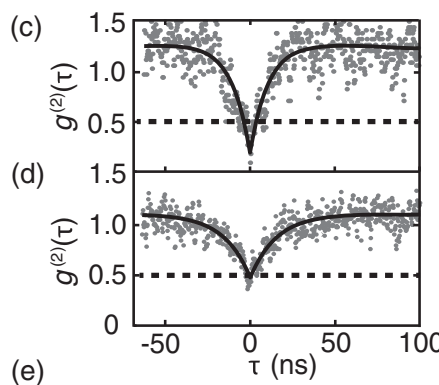

(e)

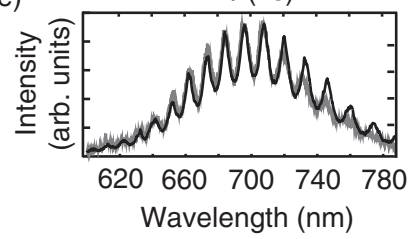

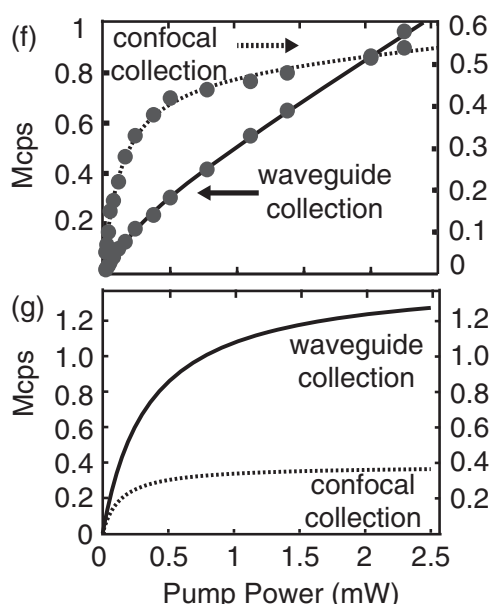

FIG. 3. (a) High-resolution confocal PL raster scan of the NV with confocal collection and (b) waveguide collection. For (b), the background is subtracted using spin-selective fluorescence [32,33]. (c) Normalized autocorrelation measurement $g^{(2)}(\tau)$ via confocal collection confirming single photon emission. (d) Cross-correlation measurement between photons collected via the confocal setup and through the waveguide confirming that the majority of photons collected through the waveguide originate from a single NV. Solid curves in (c) and (d) are fits to the data. (e) Spectrum of the emitter collected via the waveguide. The solid curve is a model with parameter values taken from measured data as detailed in Appendix E. (f) Saturation measurements acquired on the same emitter with confocal (dashed line) and waveguide (solid line) collection. (g) Fits from (f) without background and corrected for measured collection losses (see text). 
of the waveguide collection pathway is estimated to be $\sim 25 \%$ with a transmission measurement using a $633-\mathrm{nm}$ $\mathrm{HeNe}$ laser. The confocal setup is measured to have an upper-bound efficiency of $17 \%$ from the sample to the fiber-coupled detector, again from transmission of the 635-nm laser. Both signals are measured with a Si avalanche photodiode (APD) with quantum efficiency $\eta=0.65$. Without these system inefficiencies, we estimate that $0.38 \times 10^{6} \mathrm{NV}$ photons/s are collected into the objective at saturation, while $1.45 \times 10^{6} \mathrm{NV}$ photons/s are collected into one direction of the single-mode $\mathrm{SiN}$ waveguide.

The strong background seen in the optical measurements could be reduced with a distributed Bragg reflector (DBR) at the excitation laser wavelength integrated into the $\mathrm{SiN}$ waveguide near the diamond-coupling region $[34,35]$. The integration of such a filter would significantly increase the signal-to-background ratio by inhibiting fluorescence from scattered pump light in the full length of the SiN waveguide. As shown in Appendix F, transmission measurements of DBRs fabricated into single-mode $\mathrm{SiN}$ waveguides of the same design show greater than $14 \mathrm{~dB}$ of attenuation, enabling purer single photon generation.

The creation of an efficient network of entangled quantum nodes depends not only on the efficient optical coupling demonstrated above, but also on the spectral and spin properties of the quantum nodes. The precharacterization we demonstrate here is easily scaled to include these and other relevant properties.

The spectral properties of the ZPL emission determine the feasibility of various quantum protocols, including optical entanglement of two or more quantum nodes that would be necessary for the creation of a quantum network [16]. To determine the spectral properties of the NVs in our $\mu \mathrm{WGs}$, we perform photoluminescence excitation (PLE) measurements at cryogenic temperatures (18 K). Fluorescence above $650 \mathrm{~nm}$ is collected under repeated resonant laser line scans of several GHz around the ZPL of a single NV inside a $\mu \mathrm{WG}$, alternated with $100-\mu \mathrm{s} 532-\mathrm{nm}$ repumping pulses (see Appendix G).

Figures 4(a) and 4(b) show a multiscan measurement of a representative NV and an example single-line scan, respectively. Figure 4(c) shows the distribution of the measured linewidths of signal peaks in the PLE of consecutive singleline scans of $\mathrm{NV} \mathrm{C}$, with a maximally occurring linewidth of $393 \mathrm{MHz}$. This single-scan linewidth is about 30 times broader than the expected lifetime-limited linewidth of $13 \mathrm{MHz}$ for the $m_{s}=0$ transitions in bulk diamond $[36,37]$. We partially attribute this broadening to in situ spectral diffusion occurring during a single-line scan. Because of hardware limitations, we integrate 1000 times longer than necessary, continuously causing spectral diffusion $[37,38]$. This broadening could be reduced by more suitable power and timing parameters, as discussed in Appendix G. Figure 4(d) shows the mean and standard deviation of the measured single-scan linewidths of three NVs in individual $\mu$ WGs. Although these measured
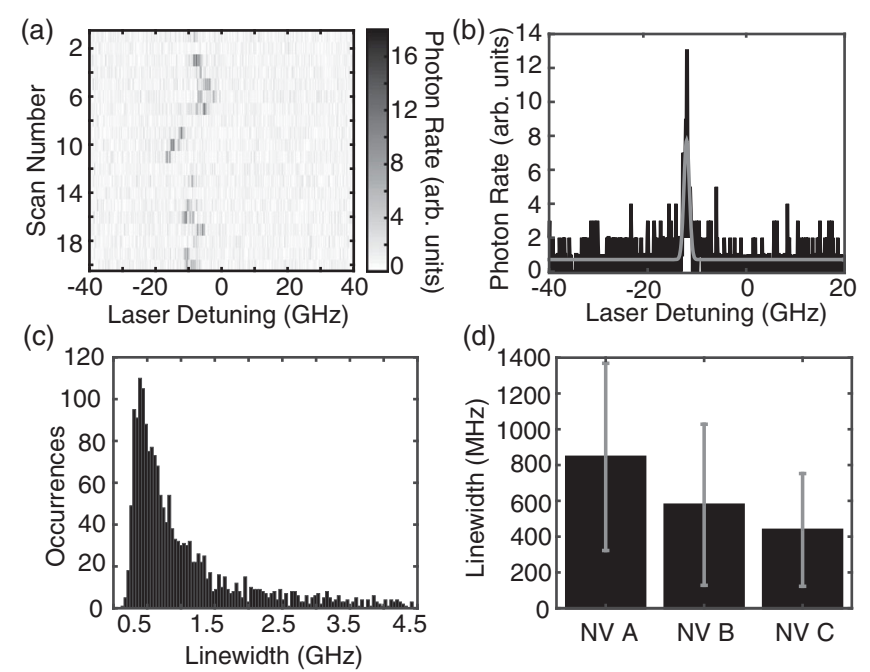

FIG. 4. (a) A typical multiscan measurement of a single, spectrometer-limited $\mathrm{NV}$ in a diamond $\mu \mathrm{WG}$ with resonant excitation and collection of the phonon sideband at cryogenic temperatures $(18 \mathrm{~K})$. (b) A single scan with a Gaussian fit showing a linewidth of $918 \mathrm{MHz}$. (c) The distribution of measured single-scan linewidths of NV C, with a maximally occurring linewidth of $393 \mathrm{MHz}$. (d) Mean and standard deviation of measured single-scan linewidths for three NVs in individual $\mu \mathrm{WGs}$.

linewidths are larger than the expected lifetime-limited linewidth, they are significantly lower than the previously reported linewidth of a NV in nanostructured diamond [39], and the dephasing times are within the time resolution of the detection equipment, allowing for measurements of twophoton interference towards distributed entanglement [16].

Another important figure of merit is the electron spin coherence time. In Fig. 5, we present the electron spin properties of the NV center in a second integrated system [WG no. 2 in Fig. 2(f)] in which $0.8 \times 10^{6}$ photons/s are collected into one direction of the waveguide at saturation. Figure 5(a) shows the transitions of the NV center with the magnetic sublevels $m_{s}=-1,0,1$, obtained using optically detected magnetic resonance (ODMR) [40]. Figure 5(b) plots the ODMR fluorescence signal collected through the waveguide under continuous-wave laser excitation with no external magnetic field.

For state manipulation, the degeneracy of the $m_{s}= \pm 1$ levels is lifted by the application of a magnetic field of $\sim 56 \mathrm{G}$ projected onto the NV axis. A Ramsey sequence, consisting of two $\pi / 2$ pulses separated by a free evolution time $\tau$, is used to probe the spin environment experienced by the NV. From this, we deduce an ensemble phase coherence time $T_{2}^{*} \simeq 2 \mu \mathrm{s}$, as seen in Fig. 5(c). We also perform Hahn-echo measurements [41], which indicate a spin coherence time of $T_{2} \simeq 120 \mu \mathrm{s}$; see Fig. 5(d). This long spin coherence time is similar to values observed in the parent diamond crystal [42]. We anticipate that using isotropically purified ${ }^{12} \mathrm{C}$ diamond together with dynamical 

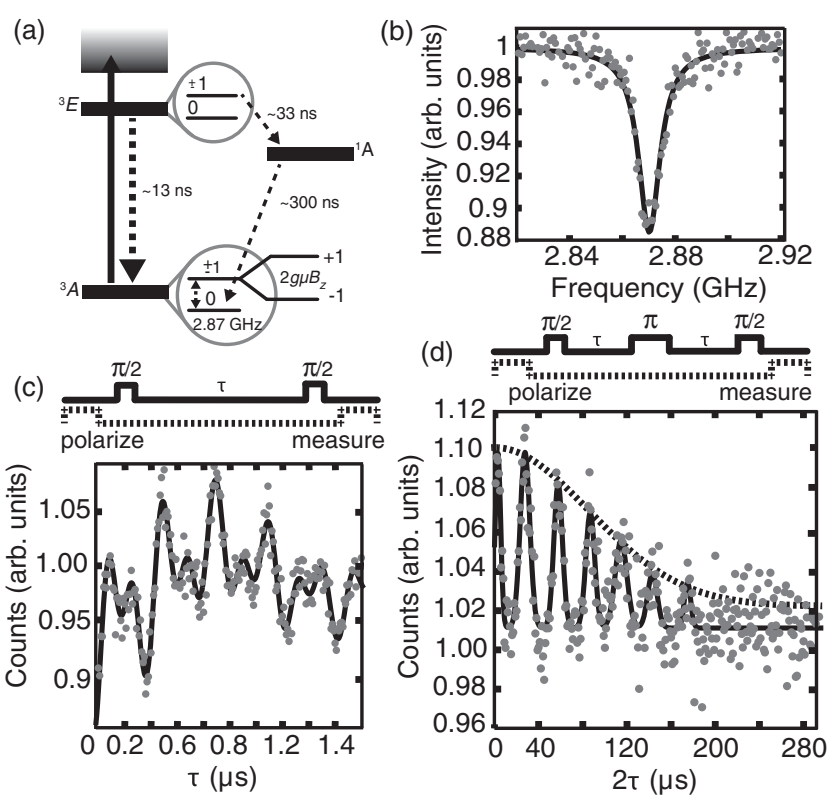

FIG. 5. (a) Level system of a NV. (b) ODMR of a NV under no magnetic field with waveguide collection. (c) The $m_{s}=0-1$ transition is driven off resonance and three Ramsey frequencies are observed due to coupling between the NV electronic spin and the host $\mathrm{N}^{14}$ nuclear spin, with a decay due to the surrounding spin bath $\left(T_{2}^{*}=2 \mu \mathrm{s}\right)$. (d) The $\pi$ and $\pi / 2$ times of an onresonance driving field are used to construct a Hahn-echo sequence to decouple the NV from the surrounding spin bath and measure $T_{2}>120 \mu \mathrm{s}$ from the exponential decay of the coherent revivals.

decoupling should enable spin coherence times in excess of tens of milliseconds [43].

In conclusion, we realize the efficient integration of four nodes-each containing a single preselected high-quality solid-state quantum memory-onto consecutive waveguides in a photonic network, overcoming the inherently low-yield fabrication process that is typical for atomlike solid-state quantum memories. The design of the diamond$\mathrm{SiN}$ interface allows for efficient coupling of photons emitted by the NV center into the single-mode $\mathrm{SiN}$ waveguide, which itself exhibits low propagation loss $(\sim 0.3 \mathrm{~dB} / \mathrm{cm})$ and enables high coupling to single-mode fiber ( $\sim 3 \mathrm{~dB}$ loss). This approach can be generalized to other systems (in particular, photonic crystal cavities optically coupled to single quantum emitters [29]) to integrate prescreened functional nodes into high-quality PICs with near unity yield, paving the way towards the scalable fabrication of on-chip quantum networks.

\section{ACKNOWLEDGMENTS}

S. .L.M. and M.W. were supported by the AFOSR Quantum Memories MURI. T.S. was supported by the Alexander von Humboldt Foundation. E. H. C. was supported by the NASA Office of the Chief Technologist's Space Technology Research Fellowship. Fabrication and experiments were supported in part by the Air Force Office of Scientific Research (AFOSR Grant No. FA9550-11-10014, supervised by Gernot Pomrenke). Research carried out in part at, and simulations were done in part at, the computing cluster of the Center for Functional Nanomaterials, Brookhaven National Laboratory, which is supported by the U.S. Department of Energy, Office of Basic Energy Sciences, under Contract No. DE-AC0298CH10886. Research also carried out in part at the Cornell NanoScale Facility, a member of the National Nanotechnology Infrastructure Network, which is supported by the National Science Foundation (Grant No. ECCS-0335765). M. L. M. and D. J. T. were supported by the DARPA Quiness program. We thank Matt Trusheim and Sen Yang for valuable discussions.

S. L. M and T. S. contributed equally to this work.

\section{APPENDIX A: FABRICATION}

We begin $\mu \mathrm{WG}$ fabrication with a $5-\mu \mathrm{m}$ ultrapure diamond slab produced by chemical vapor deposition. The diamond slab is polished with $\mathrm{Ar}$ and $\mathrm{Cl}_{2}$ down to a final thickness of $200 \mathrm{~nm}$. This membrane is patterned with oxygen plasma in a reactive ion etching chamber, using a transferrable patterned silicon membrane as an etch mask, a technique that was introduced and explained in previous publications $[29,30]$.

To create the SiN PIC, 400-nm SiN is deposited on a thermally oxidized $(\sim 4 \mu \mathrm{m}) \mathrm{Si}$ wafer in a furnace at $800^{\circ} \mathrm{C}$. After a piranha clean, ma-N negative tone resist (micro resist technology) and electron-beam lithography are used to define the waveguides. After resist development, the SiN is dry etched in a $\mathrm{CHF}_{3} / \mathrm{O}_{2}$ chemistry. The resist is then stripped in an oxygen plasma, and the wafer is then annealed at $1200^{\circ} \mathrm{C}$ for $180 \mathrm{~min}$ in an ambient nitrogen environment. $\mathrm{SiO}_{2}$ is then deposited $(\sim 3 \mu \mathrm{m})$ as a cladding material with plasma-enhanced chemical vapor deposition. To access the coupling regions, SPR positive tone resist (micro resist technology) and contact lithography are used to define windows in the $\mathrm{SiO}_{2}$. The development of the exposed resist exposes the windows, and they are etched in a buffered oxide etch until the $\mathrm{SiN}$ waveguides are fully uncovered.

\section{APPENDIX B: DIAMOND INTEGRATION}

The diamond $\mu$ WGs are detached and picked up from the initial array with a tungsten microprobe (Ted Pella) mounted to a 3-axis piezomicromanipulator and rotation stage. Each $\mu \mathrm{WG}$ is transferred to the SiN chip, which sits on a 2-axis and rotation stage. The diamond $\mu \mathrm{WG}$ is aligned to the coupling region of a $\mathrm{SiN}$ waveguide and placed in its center. Adhesion is promoted via $\mathrm{O}_{2}$ plasma cleaning of the SiN prior to placement. Optical images of the four measured $\mu \mathrm{WGs}$ placed on consecutive waveguides of the optimal design are shown in Fig. 6(a). 

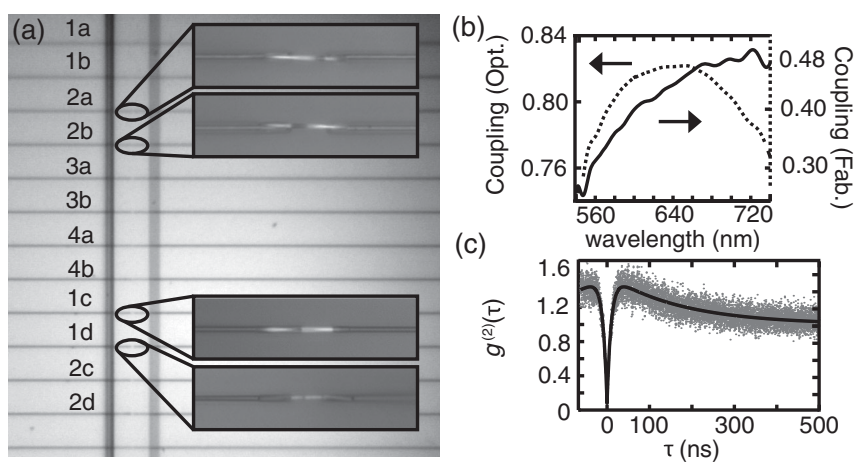

FIG. 6. (a) The coupling region of the SiN chip used with three different waveguide designs, and zoomed-in images of the four devices integrated on consecutive waveguides of the same design. (b) Simulated coupling efficiencies from the NV into the SiN of the optimized (dashed line) and fabricated (solid line) devices. (c) Autocorrelation measurement via confocal collection of a NV in a diamond $\mu \mathrm{WG}$ showing superb single photon character with antibunching down to 0.07 .

\section{APPENDIX C: SIMULATIONS}

To predict and optimize the coupling efficiency from a $\mathrm{NV}$ center in a $200 \times 200 \mathrm{~nm}$ single-mode diamond $\mu \mathrm{WG}$ to a $400 \times 400 \mathrm{~nm}$ single-mode SiN WG, we simulate our system using FDTD computations using a freely available software package (MEEP). The NV center is represented as an electric dipole placed in the center of the diamond $\mu \mathrm{WG}$, oriented perpendicular to the propagation axis of the $\mu \mathrm{WG}$ and $35^{\circ}$ off horizontal. This is consistent with a diamond slab oriented in the $\langle 100\rangle$ direction, as we use in our experiment. We place Poynting flux monitors (i) to either side of the NV, overlapping the diamond $\mu \mathrm{WG}$ before the SiN WG begins, (ii) at each end of the SiN WG, and (iii) surrounding the entire structure in order to monitor where electromagnetic power is lost. The ratio of (i) to (iii) gives the NV coupling efficiency to the diamond $\mu \mathrm{WG}$, and the ratio of (ii) to (iii) yields the total coupling efficiency of the device.

The optimized device geometry is determined by evaluating the coupling efficiency from the fundamental TE mode of the diamond $\mu \mathrm{WG}$ to the SiN WG while sweeping the diamond $\mu \mathrm{WG}$ and SiN WG taper lengths. A $2-\mu \mathrm{m}$ gap in the SiN WG is simulated to maintain high coupling from the NV into the diamond $\mu \mathrm{WG}$. The tapers are aligned such that the taper regions in $\mathrm{SiN}$ and diamond do not overlap, minimizing the photon loss due to scattering. Based on the sweep results, we choose a $\mu \mathrm{WG}$ taper length of $6 \mu \mathrm{m}$ and a SiN WG taper length of $5 \mu \mathrm{m}$. This gives a $\mu \mathrm{WG}$ that is $24 \mu \mathrm{m}$ long, and yields an overall dipole-to-WG coupling efficiency of $83 \%$ (41.5\% coupled to each side), as shown in Fig. 6(b). We also simulate the structure used in the experiments reported here, which is shorter and has tips tapered down to $100 \mathrm{~nm}$ instead of $0 \mathrm{~nm}$, which increases fabrication yield though it decreases the coupling efficiency from the diamond $\mu \mathrm{WG}$ to the $\mathrm{SiN}$ WG to $42.5 \%$, as shown in Fig. 6(b).

\section{APPENDIX D: SINGLE PHOTON MEASUREMENTS}

Photon correlation measurements are performed with a Hanbury Brown and Twiss setup. In autocorrelation measurements, the PL collected via the confocal setup is coupled to a fiber beam splitter, with the two output arms coupled to APD modules (Perkin Elmer). In cross-correlation measurements between confocal and waveguide collection channels, the two single-mode collection fibers are directly coupled into separate APDs. A histogram of arrival times is assembled using a time-correlated counting module in start-stop mode. Figure 6(c) shows a NV in an integrated nanowire exhibiting excellent single photon character with an antibunching dip down to 0.07 .

The reported single photon counts without background in Fig. 2(f) are found in two ways. One, the value of $g^{(2)}(0)$ for autocorrelation and cross-correlation measurements at the same excitation power and polarization are compared. The number of single photons is derived from the increase in this value. Two, saturation curves are taken and fit with a background term. This then allows separation of background and NV count rates at several powers, as seen in Fig. 3(g).

\section{APPENDIX E: WAVEGUIDE SPECTRA}

The PL collected through the waveguide consists of PL originating from the diamond $\mu \mathrm{WG}$ and PL caused by laser propagation through the $\mathrm{SiN}$ waveguide. The PL originating from the diamond $\mu \mathrm{WG}$ experiences an etalon effect due to the diamond $\mu \mathrm{WG}$ ends. Over the wavelength region of interest (600-780 nm), the dispersion of the waveguide mode is linear, as seen in Fig. 7(b), the group velocity dispersion in the $12-\mu \mathrm{m}$ diamond waveguide is negligible and does not affect the elatoning effect. Thus, the detected spectrum is modeled simply as

$$
\mathrm{WG}\left(\lambda_{0}\right)=a\left|\frac{(1-r)^{2} e^{-2 i \pi n L / \lambda_{0}}}{1-r^{2} e^{-4 i \pi n L / \lambda_{0}}}\right|^{2} \mathrm{NV}\left(\lambda_{0}\right)+b \mathrm{BG}\left(\lambda_{0}\right),
$$

where $\operatorname{NV}\left(\lambda_{0}\right)$ is the spectrum collected via the confocal setup [Fig. 6(d)] and $\mathrm{BG}\left(\lambda_{0}\right)$ is the spectrum of the $\mathrm{SiN}$ fluorescence collected through the waveguide [Fig. 6(d)]. $n=1.65$ is the expected effective refractive index of the diamond waveguide mode determined via eigenmode analysis of the waveguide, $L=12 \mu \mathrm{m}$ is the total length of the diamond waveguide, and $r^{2}=0.17$ due to the waveguide-air interface.

\section{APPENDIX F: INTEGRATED FILTERS}

To reduce $\mathrm{SiN}$ fluorescence in future iterations, we design, fabricate, and test DBR filters integrated into single-mode waveguides. The DBRs are designed with periodically alternating etched and full regions to give coherent reflection. 

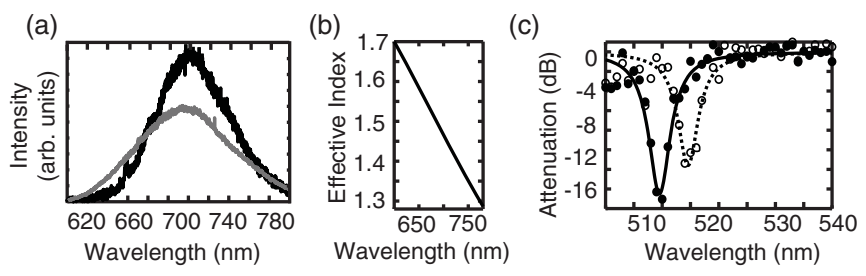

FIG. 7. (a) Normalized spectra of the NV via confocal collection (black) and the SiN waveguide fluorescence (gray) under 532-nm excitation. (b) Effective index of the waveguide mode. (c) Laser attenuation from DBR filters integrated into similar single-mode waveguides measured in transmission.

The full regions are terminated with a semicircle to take into account electron-beam resolution limits. The widths and lengths of the two regions are optimized to maximize the ratio of reflection of excitation light and transmission of a $637-n m$ ZPL signal. FDTD simulations of 60 periods of the optimized structure give greater than $40 \mathrm{~dB}$ attenuation for elliptically polarized light at the excitation wavelength with a FWHM of $\sim 30 \mathrm{~nm}$, and over 95\% ZPL transmission. As we report in the text, the first fabricated iteration of these structures results provide greater than $14 \mathrm{~dB}$ of attenuation at 515-nm excitation, a common direct-band-gap laser wavelength, which can be used to efficiently excite the NV $[44,45]$.

\section{APPENDIX G: SPECTRAL DIFFUSION MEASUREMENTS}

The reported spectral diffusion measurements are made using a resonant laser with $200 \mathrm{nW}$ of power. A $600-\mu \mathrm{s}$ green laser pulse is delivered prior to each scan to reinitialize the NV center into the $m_{s}=0$ ground state with approximately $100 \mu \mathrm{W}$. The laser frequency is scanned using a piezocontrolled grating in an external cavity diode laser (Velocity 6304, New Focus). Each step in the sweep is integrated for $5 \mathrm{~ms}$, making each sweep take 2-3 s. The step speed is limited by our hardware, and as discussed in the text, is more than 1000 times longer than necessary.

Multiple PLE peaks are contained in one scan due to spectral diffusion caused by the resonant laser during the long integration time of $5 \mathrm{~ms}$ per frequency step. To determine the fundamental linewidth, the number of PLE peaks $n$ is determined using a threshold technique, and the sum of $n$ Gaussian functions are fit to the data. Finally, the FWHM data are binned to analyze the spectral linewidth, as indicated in Fig. 4(c).

[1] C. H. Bennett and D. P. DiVincenzo, Quantum Information and Computation, Nature (London) 404, 247 (2000).

[2] H.-J. Briegel, W. Dür, J. I. Cirac, and P. Zoller, Quantum Repeaters: The Role of Imperfect Local Operations in Quantum Communication, Phys. Rev. Lett. 81, 5932 (1998).
[3] M. A. Nielsen and I. Chuang, Quantum Computation and Quantum Information (Cambridge University Press, Cambridge, England, 2000).

[4] J. I. Cirac, P. Zoller, H. J. Kimble, and H. Mabuchi, Quantum State Transfer and Entanglement Distribution among Distant Nodes in a Quantum Network, Phys. Rev. Lett. 78, 3221 (1997).

[5] H. J. Kimble, The Quantum Internet, Nature (London) 453, 1023 (2008).

[6] T. E. Northup and R. Blatt, Quantum Information Transfer Using Photons, Nat. Photonics 8, 356 (2014).

[7] P. C. Maurer, G. Kucsko, C. Latta, L. Jiang, N. Y. Yao, S. D. Bennett, F. Pastawski, D. Hunger, N. Chisholm, M. Markham, D. J. Twitchen, J. I. Cirac, and M. D. Lukin, Room-Temperature Quantum Bit Memory Exceeding One Second, Science 336, 1283 (2012).

[8] N. Bar-Gill, L. M. Pham, A. Jarmola, D. Budker, and R. L. Walsworth, Solid-State Electronic Spin Coherence Time Approaching One Second, Nat. Commun. 4, 1743 (2013).

[9] M. W. Doherty, N. B. Manson, P. Delaney, F. Jelezko, J. Wrachtrup, and L. C. L. Hollenberg, The Nitrogen-Vacancy Colour Centre in Diamond, Phys. Rep. 528, 1 (2013).

[10] P. Neumann, J. Beck, M. Steiner, F. Rempp, H. Fedder, P. R. Hemmer, J. Wrachtrup, and F. Jelezko, Single-Shot Readout of a Single Nuclear Spin, Science 329, 542 (2010).

[11] M. V. Gurudev Dutt, L. Childress, L. Jiang, E. Togan, J. Maze, F. Jelezko, A. S. Zibrov, P. R. Hemmer, and M. D. Lukin, Quantum Register Based on Individual Electronic and Nuclear Spin Qubits in Diamond, Science 316, 1312 (2007).

[12] G. Waldherr, Y. Wang, S. Zaiser, M. Jamali, T. SchulteHerbrüggen, H. Abe, T. Ohshima, J. Isoya, J. F. Du, P. Neumann et al., Quantum Error Correction in a Solid-State Hybrid Spin Register, Nature (London) 506, 204 (2014).

[13] L. I. Childress, J. M. Taylor, A. Sorensen, and M. D. Lukin, Fault-Tolerant Quantum Repeaters with Minimal Physical Resources and Implementations Based on Single-Photon Emitters, Phys. Rev. A 72, 052330 (2005).

[14] K. Nemoto, M. Trupke, S. J. Devitt, A. M. Stephens, B. Scharfenberger, K. Buczak, T. Nöbauer, M. S. Everitt, J. Schmiedmayer, and W. J. Munro, Photonic Architecture for Scalable Quantum Information Processing in Diamond, Phys. Rev. X 4, 031022 (2014).

[15] W. Pfaff, B. Hensen, H. Bernien, S. B. van Damm, M. S. Blok, T. H. Taminiau, M. J. Tiggelman, R. N. Schouten, M. Markham, D. J. Twitchen, and R. Hanson, Unconditional Quantum Teleportation between Distant Solid-State Quantum Bits, Science 345, 532 (2014).

[16] H. Bernien, B. Hensen, W. Pfaff, G. Koolstra, M. S. Blok, L. Robledo, T. H. Taminiau, M. Markham, D. J. Twitchen, L. Childress, and R. Hanson, Heralded Entanglement between Solid-State Qubits Separated by Three Metres, Nature (London) 497, 86 (2013).

[17] J. S. Levy, A. Gondarenko, M. A. Foster, A. C. TurnerFoster, A. L. Gaeta, and M. Lipson, CMOS-Compatible Multiple-Wavelength Oscillator for On-Chip Optical Interconnects, Nat. Photonics 4, 37 (2010).

[18] D. J. Moss, R. Morandotti, A. L. Gaeta, and M. Lipson, New CMOS-Compatible Platforms Based on Silicon Nitride and Hydex for Nonlinear Optics, Nat. Photonics 7, 597 (2013). 
[19] S. Pezzagna, B. Naydenov, F. Jelezko, J. Wrachtrup, and J. Meijer, Creation Efficiency of Nitrogen-Vacancy Centres in Diamond, New J. Phys. 12, 065017 (2010).

[20] I. Bayn, E. H. Chen, M. E. Trusheim, L. Li, T. Schröder, O. Gaathon, M. Lu, A. Stein, M. Liu, K. Kisslinger, H. Clevenson, and D. Englund, Generation of Ensembles of Individually Resolvable Nitrogen Vacancies Using Nanometer-Scale Apertures in Ultrahigh-Aspect Ratio Planar Implantation Masks, Nano Lett. 15, 1751 (2015).

[21] B. J. M. Hausmann, B. Shields, Q. Quan, P. Maletinsky, M. McCutcheon, J. T. Choy, T. M. Babinec, A. Kubanek, A. Yacoby, M. D. Lukin, and M. Loncar, Integrated Diamond Networks for Quantum Nanophotonics, Nano Lett. 12, 1578 (2012).

[22] B. J. M. Hausmann, I. Bulu, P. B. Deotare, M. McCutcheon, V. Venkatraman, M. Markham, D. J. Twitchen, and M. Loncar, Integrated High-Quality Factor Optical Resonators in Diamond, Nano Lett. 13, 5791 (2013).

[23] I. Bayn, S. Mouradian, L. Li, J. A. Goldstein, T. Schröder, J. Zheng, E. H. Chen, O. Gaathon, M. Lu, A. Stein, C. A. Ruggiero, J. Salzman, R. Kalish, and D. Englund, Fabrication of Triangular Nanobeam Waveguide Networks in Bulk Diamond using Single-Crystal Silicon Hard Masks, Appl. Phys. Lett. 105, 211101 (2014).

[24] O. Benson, Assembly of Hybrid Photonic Architectures from Nanophotonic Constituents, Nature (London) 480, 193 (2011).

[25] C. Santori, P. E. Barclay, K.-M. C. Fu, R. G. Beausoleil, S. Spillane, and M. Fisch, Nanophotonics for Quantum Optics Using Nitrogen-Vacancy Centers in Diamond, Nanotechnology 21, 274008 (2010).

[26] M. Barth, S. Schietinger, T. Schröder, T. Aichele, and O. Benson, Controlled Coupling of NV Defect Centers to Plasmonic and Photonic Nanostructures, J. Lumin. 130, 1628 (2010).

[27] T. Schröder, A. W. Schell, G. Kewes, T. Aichele, and O. Benson, Fiber-Integrated Diamond-Based Single Photon Source, Nano Lett. 11, 198 (2011).

[28] A. W. Schell, G. Kewes, T. Schröder, J. Wolters, T. Aichele, and O. Benson, A Scanning Probe-Based Pick-and-Place Procedure for Assembly of Integrated Quantum Optical Hybrid Devices, Rev. Sci. Instrum. 82, 073709 (2011).

[29] L. Li, T. Schröder, E. H. Chen, M. Walsh, I. Bayn, J. Goldstein, O. Gaathon, M. E. Trusheim, M. Lu, J. Mower, M. Cotlet, M. L. Markham, D. J. Twitchen, and D. Englund, Coherent Spin Control of a Nanocavity-Enhanced Qubit in Diamond, Nat. Commun. 6, 6173 (2015).

[30] L. Li, I. Bayn, M. Lu, C.-Y. Nam, T. Schröder, A. Stein, N. C. Harris, and D. Englund, Nanofabrication on Unconventional Substrates Using Transferred Hard Masks, Sci. Rep. 5, 7802 (2015).

[31] L. Li, M. Trusheim, O. Gaathon, K. Kisslinger, C.-J. Cheng, M. Lu, D. Su, X. Yao, H.-C. Huang, I. Bayn, A. Wolcott, R. M. Osgood, Jr., and D. Englund, Reactive Ion Etching: Optimized Diamond Thin-Membrane Fabrication for Transmission Electron Microscopy, J. Vac. Sci. Technol. B 31, 06FF01 (2013).

[32] E. H. Chen, O. Gaathon, M. E. Trusheim, and D. Englund, Wide-Field Multispectral Super-resolution Imaging Using
Spin-Dependent Fluorescence in Nanodiamonds, Nano Lett. 13, 2073 (2013).

[33] S. K. Sarkar, A. Bumb, X. Wu, K. A. Sochacki, P. Kellman, M. W. Brechbiel, and K. C. Neuman, Wide-Field In Vivo Background Free Imaging by Selective Magnetic Modulation of Nanodiamond Fluorescence, Biomed. Opt. Express 5, 1190 (2014).

[34] N. C. Harris, D. Grassani, A. Simbula, M. Pant, M. Galli, T. Baehr-Jones, M. Hochberg, D. Englund, D. Bajoni, and C. Galland, Integrated Source of Spectrally Filtered Correlated Photons for Large-Scale Quantum Photonic Systems, Phys. Rev. X 4, 041047 (2014).

[35] X. Wang, W. Shi, H. Yun, S. Grist, N. A. F. Jaeger, and L. Chrostowski, Narrow-Band Waveguide Bragg Gratings on SOI Wafers with CMOS-Compatible Fabrication Process, Opt. Express 20, 15547 (2012).

[36] L. Robledo, H. Bernien, T. van der Sar, and R. Hanson, Spin Dynamics in the Optical Cycle of Single Nitrogen-Vacancy Centres in Diamond, New J. Phys. 13, 025013 (2011).

[37] Y. Chu, N. P. de Leon, B. J. Shields, B. Hausmann, R. Evans, E. Togan, M. J. Burek, M. Markham, A. Stacey, A. S. Zibrov, A. Yacoby, D. J. Twitchen, M. Loncar, H. Park, P. Maletinsky, and M. D. Lukin, Coherent Optical Transitions in Implanted Nitrogen Vacancy Centers, Nano Lett. 14, 1982 (2014).

[38] J. Wolters, N. Sadzak, A. W. Schell, T. Schröder, and O. Benson, Measurement of the Ultrafast Spectral Diffusion of the Optical Transition of Nitrogen Vacancy Centers in Nano-Size Diamond Using Correlation Interferometry, Phys. Rev. Lett. 110, 027401 (2013).

[39] A. Faraon, C. Santori, Z. Huang, V. M. Acosta, and R. G. Beausoleil, Coupling of Nitrogen-Vacancy Centers to Photonic Crystal Cavities in Monocrystalline Diamond, Phys. Rev. Lett. 109, 033604 (2012).

[40] N. B. Manson, J. P. Harrison, and M. J. Sellars, NitrogenVacancy Center in Diamond: Model of the Electronic Structure and Associated Dynamics, Phys. Rev. B 74, 104303 (2006).

[41] R Hanson, O. Gywat, and D. D. Awschalom, RoomTemperature Manipulation and Decoherence of a Single Spin in Diamond, Phys. Rev. B 74, 161203 (2006).

[42] J. Hodges, L. Li, E. Chen, M. Trusheim, S. Allegri, M. Lu, and D. Englund, Ultralong Spin Coherence Time 100 nm Diamond Membranes, New J. Phys. 14, 093004 (2012).

[43] G. Balasubramanian, P. Neumann, D. Twitchen, M. Markham, R. Kolesov, N. Mizuochi, J. Isoya, J. Achard, J. Beck, J. Tissler, V. Jacques, P. R. Hemmer, F. Jelezko, and J. Wrachtrup, Ultralong Spin Coherence Time in Isotopically Engineered Diamond, Nat. Mater. 8, 383 (2009).

[44] N. Aslam, G. Waldherr, P. Neumann, F. Jelezko, and J. Wrachtrup, Photo-induced Ionization Dynamics of the Nitrogen Vacancy Defect in Diamond Investigated by Single-Shot Charge State Detection, New J. Phys. 15, 013064 (2013).

[45] T. Oeckinghaus, R. Stöhr, R. Kolesov, J. Tisler, F. Reinhard, and J. Wrachtrup, A Compact, Diode Laser Based Excitation System for Microscopy of NV Centers, Rev. Sci. Instrum. 85, 073101 (2014). 